\title{
The Nonlinear Dynamic Behaviors of Electrostatic Micro-Tweezers
}

\author{
Bo-Wun Huang ${ }^{1, *}$, Chao-Jung Chen ${ }^{2}$ and Jao-Hwa Kuang ${ }^{3}$ \\ ${ }^{1}$ Department of Mechanical Engineering, Cheng Shiu University, Kaohsiung, 83347, \\ Taiwan. \\ ${ }^{2}$ Quanta Computer Inc., Taiwan. \\ ${ }^{3}$ Department of Mechanical and Electro-Mechanical Engineering, National Sun \\ Yat-Sen University, Kaohsiung, 80424, Taiwan.
}

Received 13 March 2017; Accepted (in revised version) 1 June 2017

\begin{abstract}
This work addresses the nonlinear dynamic behavior of different electrostatic micro-tweezers, a micro electric actuator. This actuator, a cantilever beam electrostatic micro-tweezers, has been extensively used in micro-electro-mechanical systems (MEMS). The importance of micro electric actuators manufactured is higher than the other part of MEMS since it is the power source of the entire micro-electro-mechanical systems. In actual operation, the instability and bad dynamic characteristics of the electric actuators will cause larger displacement mobility error, such as transport behavior and response procedures failure, etc., and even damage the micro-electro-mechanical systems. To improve the actuator dynamic displacement accuracy, the dynamic behavior in the electric actuator system must be studied, especially for nonlinear dynamic behavior of system. In this work, the differential quadrature method (DQM) was employed to solve the problem of nonlinearity in the equation of motion. The results reveal that the proposed DQM model can be used to simulate the nonlinear behavior of the micro-tweezers efficiently Micro-tweezers of various shapes were studied to examine the feasibility of applying the DQM in analyzing their nonlinear responses. The simulated results agree very closely with the calculated and experimental data in the literature.
\end{abstract}

AMS subject classifications: 35G50, 37M05, 65E05

Key words: Microelectromechanical, pull-in, Differential Quadrature Method (DQM), MEMS, electrostatic, micro-tweezers.

\section{Introduction}

Cantilever beam electrostatic micro-tweezers have been extensively used in microelectromechanical systems (MEMS). Some investigations [1,2] developed and simulated

${ }^{*}$ Corresponding author. Email addresses: huangbw@gcloud.csu.edu.tw (B.-W. Huang), james-chen@quantatw.com (C.-J. Chen), kuang@faculty.nsysu.edu.tw (J.-H. Kuang) 
the chemical vapor deposition (CVD) of tungsten with isolated silicon dioxide to form the microelectromechanical structures of micro-tweezers. In [3], a combination exterior boundary-element method (BEM) for determining the electrostatic force and a finiteelement method (FEM) for determining the elasticity of beam were proposed to quantify the coupling between the electrostatic force and the elastic deformation of the microtweezers.

Some three-dimensional methods have been developed for evaluating the nonlinear behavior of electrostatic devices. A 3-D structural simulator, OYSTER, was proposed in [5] to address 3-D geometric effects in the fabricated process. The importance of the electrostatic effect in the design and modeling of micro-actuators was discussed in [6]. Senturia et al. $[7,8]$ developed MEMS systems using the ABAQUS package and the BEM program, FASTCAP, for electrostatic analysis to solve the nonlinear-coupled equations.

The pull-in behavior of different beam structures and pressure sensors [9] was elucidated using three models, i.e. the lumped parallel-plate spring model, the onedimensional analytical model and the three-dimensional finite-element model. In [10], the pull-in instability phenomena of different beam actuators was used to determine the corresponding MEMS material parameters.

In 1997, Legtenberg et al. [11] employed the Rayleigh-Ritz method to examine the large displacement characteristics of cantilever beam type actuators. In [12], the effect of squeeze-film damping on the dynamics responses of a nonlinear actuator was studied using three-dimensional MEMCAD and FEM programs in [12]. In [13], leveraged bending and strain-stiffening methods were presented to enlarge the distance traveled before the electrostatic actuator is pulled in. The SOI (silicon-on-insulator) was used to fabricate the micro-actuators and micro-grippers, which provide the advantages of being stable and requiring a few fabrication steps [14]. In [15], SU8 photoepoxy with its excellent aspect ratio and attainable film thickness was used to fabricate shape memory alloy and actuated micro-grippers. Researchers $[16,17]$ focus the new applications of micro grippers on biological and micro robot. These studies developed to show the feasibility of micro robots and biological science. Recently, the electro thermal micro gripper was attracted to some investigations $[18,19]$.

In 1972, Bellman et al. [20] first proposed the differential quadrature method (DQM) to solve nonlinear partial equations. Before then, DQM had been widely employed to calculate the flexural vibration of a geometrically nonlinear beam [21], and to perform static and free vibration analyses of beams and plates [22]. Bert and Malik [23] used the differential quadrature method to evaluate the dynamic characteristics of various tapered rectangular plates. Bert [24] further applied particular boundary conditions to the differential quadrature weighting coefficient matrix, without applying the so-called $\delta$ technique. Chen and Zhong [25] introduced Hadamard product to derive the weighting matrices for differential quadrature and differential cubature solutions to nonlinear differential and integro-differential equations. The Hadamard and SJT product approach was proposed in reference [26] to simplify the nonlinear equations and minimize the effort of evaluating the Jacobian derivative matrix by the Newton-Raphson method. The 
generalized differential quadrature method (GDQM) was used to reduce the nonlinear boundary-initial-value problem to nonlinear ordinary differential equations [27]. In [28], the generalized differential quadrature rule (GDQR) was presented to solve initial-value differential equations of the second to fourth order. An iterative method based on the differential quadrature rules was recently further proposed for simulating nonlinearly coupled oscillators [29]. The generalized differential quadrature rule (GDQR) was also applied to both spatial and temporal dimensions simultaneously, without the application of any classical method to the temporal dimension to solve the force vibration problem [30].

In this study, the differential quadrature method is applied to formulate the nonlinear deflection equation for an electrostatic micro-tweezers in discrete form. The proposed model incorporates the effects of position-dependent electrostatic force and the mechanical restoring force. Additionally, the pull-in effects associated with various micro-tweezers were examined.

\section{Models of electrostatic micro-tweezers}

\subsection{Analysis model}

Fig. 1 schematically depicts cantilever beam micro-tweezers that consist of two individual shaped micro-arms. The application of an external voltage, $V$, between these two deformable micro-arm beams generates a position-dependent electrostatic pressure, $P_{e}(x, V)$, which brings the two deformable micro-arms toward each other. This distributed electrostatic pressure is approximately proportional to the inverse of the square of the gap between them, so a parallel micro-arm beams approximation [9] can be applied. When the applied driving voltage exceeds a critical pull-in voltage, the two cantilever micro-arm beams are suddenly pulled together.

Fig. 1 reveals that the nonlinear differential equations for shaped cantilever beam micro-tweezers are as given in $[9,11]$

$$
\frac{d^{2}}{d x^{2}}\left[E I(x) \frac{d^{2} y(x)}{d x^{2}}\right]=P_{e}(x, V)=\frac{1}{2}\left[\frac{\varepsilon_{0} b V^{2}}{4\left[g_{0}+x \sin \theta-y\right]^{2}}\right] .
$$

The corresponding non-dimensionalized form is shown to be given by

$$
\bar{h}^{3}(\bar{x}) \frac{d^{4} \bar{y}(\bar{x})}{d \bar{x}^{4}}+2 \frac{d \bar{h}^{3}(\bar{x})}{d \bar{x}} \frac{d^{3} \bar{y}(\bar{x})}{d \bar{x}^{3}}+\frac{d^{2} \bar{h}^{3}(\bar{x})}{d \bar{x}^{2}} \frac{d^{2} \bar{y}(\bar{x})}{d \bar{x}^{2}}=\bar{F}\left[\frac{1}{4\left[\bar{g}_{0}+\bar{x} \sin \theta-\bar{y}\right]^{2}}\right],
$$

with $\bar{x}=x / L_{b}, \bar{b}=b / L_{b}, g_{0}=d_{0} / 2, \bar{g}_{0}=g_{0} / L_{b}, \bar{F}=3 \varepsilon_{0} V^{2} /\left(2 E L_{b}^{2}\right)$, and $L_{b}$ is the length of the micro-arm. Moreover, $\bar{h}(\bar{x})=\bar{h}_{0}\left[1+\left(\frac{\bar{h}_{1}}{\bar{h}_{0}}-1\right)\left(\frac{\bar{x}}{\cos \theta}\right)^{m}\right]$ is the shape of the micro-beam; $\bar{h}_{0}=h_{0} / L_{b}$ and $\bar{h}_{1}=h_{1} / L_{b}$ are the non-dimensional beam thicknesses at $\bar{x}=0$ and $\bar{x}=1$, and $m$ is the order of the shape profile. 

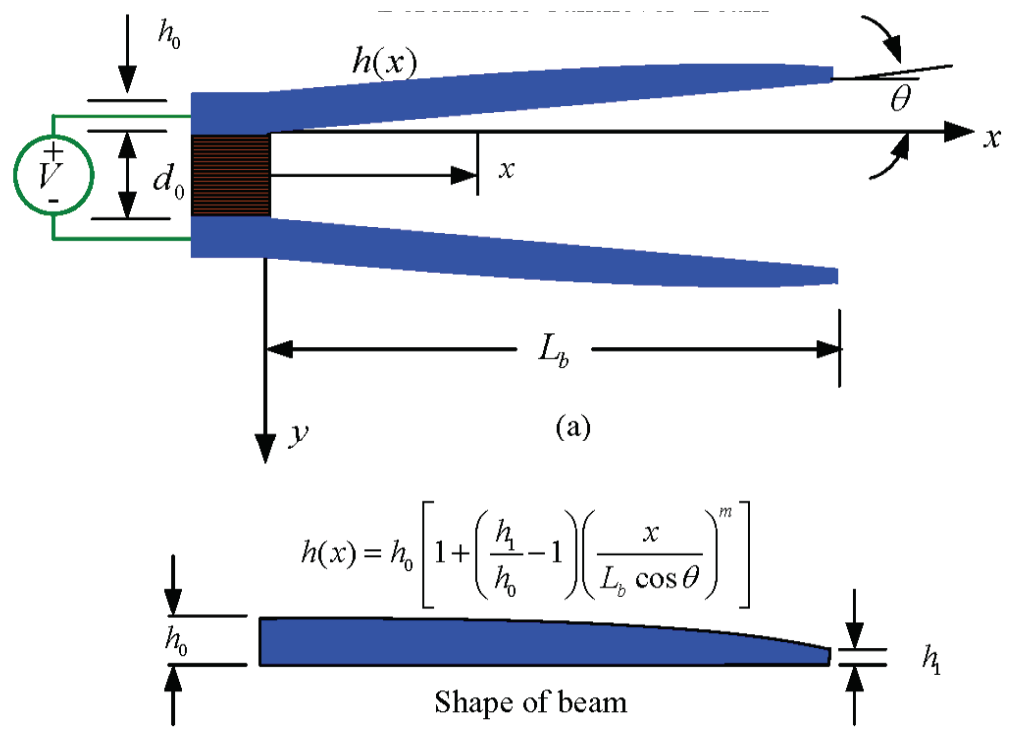

(b)

Figure 1: The cantilever beam-type micro-tweezers.

In Eq. (2.1), $E$ is the Young's modulus of micro-arm material; $I$ is the moment of inertia associated with the cross-section of the beam; $\varepsilon_{0}$ is the dielectric constant of air; $b$ is the width of the beam, and $d_{0}$ is the initial gap between the two cantilever micro-arm beams at the fixed end $(\bar{x}=0)$. The corresponding non-dimensional boundary conditions are

$$
\begin{array}{lc}
\bar{y}(\bar{x})=0, \quad \bar{y}^{\prime}(\bar{x})=0, \quad \text { at } \quad \bar{x}=0, \\
\bar{y}^{\prime \prime}(\bar{x})=0, \quad \bar{y}^{\prime \prime \prime}(\bar{x})=0, \quad \text { at } \bar{x}=1 .
\end{array}
$$

\subsection{Differential Quadrature Method (DQM)}

In this work, the differential quadrature method (DQM) [20-30] is used to formulate the nonlinear differential equation, Eq. (2.2), in discrete form. The fundamental theory of the DQM is that the different order partial derivatives of a function at a given point can be approximated using a weighted sum of function values at all discrete points in that domain. With reference to the DQM, the $r^{\text {th }}$ order derivative of a function $y(x)$ at a given point $x_{i}$ can be expressed using $N$ weighted function values at various sampling points [26]:

$$
\left.\frac{d^{r} y(x)}{d x^{r}}\right|_{x=x_{i}}=\sum_{j=1}^{N} w_{i j}^{(r)} y_{j}, \quad \text { for } i=1,2, \cdots, N \text {, }
$$


where $y_{j}=y\left(x_{j}\right)$ and $w_{i j}^{(r)}$ represents the corresponding weighting coefficients. The weighting coefficients of the first derivatives can be obtained from:

$$
\begin{array}{rlrl}
w_{i j}^{(1)} & =\frac{1}{x_{j}-x_{i}} \prod_{k \neq i, k \neq j, k=1}^{N} \frac{x_{i}-x_{k}}{x_{j}-x_{k}}, & & i=1,2, \cdots, N \quad \text { and } j=1,2, \cdots, N, \\
w_{i i}^{(1)}=\sum_{k \neq i}^{N} \frac{1}{x_{i}-x_{k}}, & i=1,2, \cdots, N .
\end{array}
$$

The weighting coefficients of the higher-order derivatives are obtained by matrix multiplication [26]:

$$
w_{i j}^{(2)}=\sum_{k=1}^{N} w_{i k}^{(1)} w_{k j}^{(1)}, \quad w_{i j}^{(3)}=\sum_{k=1}^{N} w_{i k}^{(1)} w_{k j}^{(2)}, \quad w_{i j}^{(4)}=\sum_{k=1}^{N} w_{i k}^{(1)} w_{k j}^{(3)}, \quad \text { for } i, j=1,2, \cdots, N .
$$

This study employs the Newton-Raphson method to solve the set of nonlinear equations resulting from the DQM equations. For convenience, the Hadamard product [25] and the SJT matrix product [26] are used. These two products enable the Frechet derivative matrix of the Newton-Raphson method to be calculated using a more efficient and explicit procedure. The Hadamard product and the SJT product are defined as follows:

Definition 2.1. Let matrices $[A]=\left[a_{i j}\right]$ and $[B]=\left[b_{i j}\right] \in C^{N \times M}$; the Hadamard product of matrices is defined as $[A] \circ[B]=\left[a_{i j} b_{i j}\right] \in C^{N \times M}$. Accordingly, $[A]^{\circ q}=\left[a_{i j}^{q}\right] \in C^{N \times M}$.

Definition 2.2. Let matrix $[A]=\left[a_{i j}\right] \in C^{N \times M}$ and vector $\{B\}=\left\{b_{i j}\right\} \in C^{N \times 1}$, such that the SJT product of the matrix and the vector is defined as $[A] \diamond\{B\}=\left[a_{i j} b_{j}\right] \in C^{N \times M}$.

From Definition 2.1, the Hadamard product is consistent the theorem $[A] \circ[B]=[B] \circ$ $[A]$ and $([A]+[B]) \circ[C]=[A] \circ[C]+[B] \circ[C]$.

The Newton-Raphson method is a fundamental numerical method for solving the set of nonlinear equations, obtained from the DQM formulations of the nonlinear differential equations. One of the most time-consuming tasks associated with this method is calculating the Frechet derivative matrix. Chen et al. [26] proposed that the post-multiplication SJT product of the matrix and vector should be used to simply the computation of the Frechet matrix.

Using the SJT product enables the analytical solution of Frechet derivative matrix to be obtained. The effort required for computing one SJT product involves only an $\mathcal{O}\left(n^{2}\right)$ scalar multiplication and so the computational cost is minimal [25].

The nonlinear quadratic operator $f_{, x} f_{, y}$ in the DQM can be expressed in Hadamard product form as $f_{, x} f_{, y}=\left(w_{x} \vec{f}\right) \circ\left(w_{y} \vec{f}\right)$ [26], where $\vec{f}$ is a vector. Furthermore, $f_{, x}$ and $f_{, y}$ represent the partial derivative vectors of the function in the $x$ and $y$ directions, respectively. Meanwhile, $w_{x}$ and $w_{y}$ are the weighting matrices of DQM for the first derivatives 
in the $x$ and $y$ directions, respectively. The corresponding non-dimensionalized form of Eq. (2.2) can be shown to be

$$
\begin{aligned}
& \bar{h}^{3}(\bar{x}) \frac{d^{4} \bar{y}(\bar{x})}{d \bar{x}^{4}}+2 \frac{d \bar{h}^{3}(\bar{x})}{d \bar{x}} \frac{d^{3} \bar{y}(\bar{x})}{d \bar{x}^{3}}+\frac{d^{2} \bar{h}^{3}(\bar{x})}{d \bar{x}^{2}} \frac{d^{2} \bar{y}(\bar{x})}{d \bar{x}^{2}} \\
= & \bar{F}\left[\frac{1}{\left[\bar{g}_{0}+\bar{x} \sin \theta\right]^{2}}+\frac{2 \bar{y}(\bar{x})}{\left[\bar{g}_{0}+\bar{x} \sin \theta\right]^{3}}+\frac{3 \bar{y}^{2}(\bar{x})}{\left[\bar{g}_{0}+\bar{x} \sin \theta\right]^{4}}+\frac{4 \bar{y}^{3}(\bar{x})}{\left[\bar{g}_{0}+\bar{x} \sin \theta\right]^{5}}+\cdots\right] .
\end{aligned}
$$

The corresponding non-dimensional boundary conditions are

$$
\bar{y}(\bar{x})=\bar{y}^{\prime}(\bar{x})=0, \quad \text { at } \quad \bar{x}=0 \quad \text { and } \quad \bar{y}^{\prime \prime}(\bar{x})=\bar{y}^{\prime \prime \prime}(\bar{x})=0, \quad \text { at } \quad \bar{x}=1 .
$$

The weighting coefficient matrices incorporate the set of boundary conditions. Therefore, the weighting coefficient matrices in this case can be shown to be

$$
\begin{aligned}
& w_{i j}^{(1)}=\left[\begin{array}{ccccc}
0 & 0 & \cdots & 0 & 0 \\
0 & w_{22}^{(1)} & \cdots & w_{2, N-1}^{(1)} & w_{2, N}^{(1)} \\
\vdots & \vdots & \ddots & \vdots & \vdots \\
0 & w_{N-1,2}^{(1)} & \cdots & w_{N-1, N-1}^{(1)} & w_{N-1, N}^{(1)} \\
0 & w_{N, 2}^{(1)} & \cdots & w_{N, N-1}^{(1)} & w_{N, N}^{(1)}
\end{array}\right] \\
& w_{i j}^{(2)}=\left[\begin{array}{ccccc}
w_{11}^{(1)} & w_{12}^{(1)} & \cdots & w_{1, N-1}^{(1)} & w_{1, N}^{(1)} \\
w_{21}^{(1)} & w_{22}^{(1)} & \cdots & w_{2, N-1}^{(1)} & w_{2, N}^{(1)} \\
\vdots & \vdots & \ddots & \vdots & \vdots \\
w_{N-1,1}^{(1)} & w_{N-1,2}^{(1)} & \cdots & w_{N-1, N-1}^{(1)} & w_{N-1, N}^{(1)} \\
0 & 0 & \cdots & 0 & 0
\end{array}\right]\left[\begin{array}{ccccc}
0 & 0 & \cdots & 0 & 0 \\
0 & w_{22}^{(1)} & \cdots & w_{2, N-1}^{(1)} & w_{2, N}^{(1)} \\
\vdots & \vdots & \ddots & \vdots & \vdots \\
0 & w_{N-1,2}^{(1)} & \cdots & w_{N-1, N-1}^{(1)} & w_{N-1, N}^{(1)} \\
0 & w_{N, 2}^{(1)} & \cdots & w_{N, N-1}^{(1)} & w_{N, N}^{(1)}
\end{array}\right], \\
& w_{i j}^{(3)}=\left[\begin{array}{ccccc}
w_{11}^{(1)} & w_{12}^{(1)} & \cdots & w_{1, N-1}^{(1)} & w_{1, N}^{(1)} \\
w_{21}^{(1)} & w_{22}^{(1)} & \cdots & w_{2, N-1}^{(1)} & w_{2, N}^{(1)} \\
\vdots & \vdots & \ddots & \vdots & \vdots \\
w_{N-1,1}^{(1)} & w_{N-1,2}^{(1)} & \cdots & w_{N-1, N-1}^{(1)} & w_{N-1, N}^{(1)} \\
0 & 0 & \cdots & 0 & 0
\end{array}\right]\left[\begin{array}{ccccc}
w_{11}^{(1)} & w_{12}^{(1)} & \cdots & w_{1, N-1}^{(1)} & w_{1, N}^{(1)} \\
w_{21}^{(1)} & w_{22}^{(1)} & \cdots & w_{2, N-1}^{(1)} & w_{2, N}^{(1)} \\
\vdots & \vdots & \ddots & \vdots & \vdots \\
w_{N-1,1}^{(1)} & w_{N-1,2}^{(1)} & \cdots & w_{N-1, N-1}^{(1)} & w_{N-1, N}^{(1)} \\
0 & 0 & \cdots & 0 & 0
\end{array}\right] \\
& {\left[\begin{array}{ccccc}
0 & 0 & \cdots & 0 & 0 \\
0 & w_{22}^{(1)} & \cdots & w_{2, N-1}^{(1)} & w_{2, N}^{(1)} \\
\vdots & \vdots & \ddots & \vdots & \vdots \\
0 & w_{N-1,2}^{(1)} & \cdots & w_{N-1, N-1}^{(1)} & w_{N-1, N}^{(1)} \\
0 & w_{N, 2}^{(1)} & \cdots & w_{N, N-1}^{(1)} & w_{N, N}^{(1)}
\end{array}\right]}
\end{aligned}
$$




$$
\begin{aligned}
w_{i j}^{(4)}= & {\left[\begin{array}{ccccc}
w_{11}^{(1)} & w_{12}^{(1)} & \cdots & w_{1, N-1}^{(1)} & w_{1, N}^{(1)} \\
w_{21}^{(1)} & w_{22}^{(1)} & \cdots & w_{2, N-1}^{(1)} & w_{2, N}^{(1)} \\
\vdots & \vdots & \ddots & \vdots & \vdots \\
w_{N-1,1}^{(1)} & w_{N-1,2}^{(1)} & \cdots & w_{N-1, N-1}^{(1)} & w_{N-1, N}^{(1)} \\
w_{N, 1}^{(1)} & w_{N, 2}^{(1)} & \cdots & w_{N, N-1}^{(1)} & w_{N, N}^{(1)}
\end{array}\right]\left[\begin{array}{ccccc}
w_{11}^{(1)} & w_{12}^{(1)} & \cdots & w_{1, N-1}^{(1)} & w_{1, N}^{(1)} \\
w_{21}^{(1)} & w_{22}^{(1)} & \cdots & w_{2, N-1}^{(1)} & w_{2, N}^{(1)} \\
\vdots & \vdots & \ddots & \vdots & \vdots \\
w_{N-1,1}^{(1)} & w_{N-1,2}^{(1)} & \cdots & w_{N-1, N-1}^{(1)} & w_{N-1, N}^{(1)} \\
0 & 0 & \cdots & 0 & 0
\end{array}\right] } \\
& {\left[\begin{array}{ccccc}
w_{11}^{(1)} & w_{12}^{(1)} & \cdots & w_{1, N-1}^{(1)} & w_{1, N}^{(1)} \\
w_{21}^{(1)} & w_{22}^{(1)} & \cdots & w_{2, N-1}^{(1)} & w_{2, N}^{(1)} \\
\vdots & \vdots & \ddots & \vdots & \vdots \\
w_{N-1,1}^{(1)} & w_{N-1,2}^{(1)} & \cdots & w_{N-1, N-1}^{(1)} & w_{N-1, N}^{(1)} \\
0 & 0 & \cdots & 0 & 0
\end{array}\right]\left[\begin{array}{ccccc}
0 & 0 & \cdots & 0 & 0 \\
0 & w_{22}^{(1)} & \cdots & w_{2, N-1}^{(1)} & w_{2, N}^{(1)} \\
\vdots & \vdots & \ddots & \vdots & \vdots \\
0 & w_{N-1,2}^{(1)} & \cdots & w_{N-1, N-1}^{(1)} & w_{N-1, N}^{(1)} \\
0 & w_{N, 2}^{(1)} & \cdots & w_{N, N-1}^{(1)} & w_{N, N}^{(1)}
\end{array}\right] }
\end{aligned}
$$

The DQM can be used to write the basic iterative equation in the Hadamard and SJT products as

$$
\begin{aligned}
\varphi\{\vec{y}\}= & \bar{h}^{3}(\bar{x}) \circ\left(w_{i j}^{(4)} \vec{y}\right)+2 \frac{d \bar{h}^{3}(\bar{x})}{d \bar{x}} \circ\left(w_{i j}^{(3)} \vec{y}\right)+\frac{d^{2} \bar{h}^{3}(\bar{x})}{d \bar{x}^{2}} \circ\left(w_{i j}^{(2)} \vec{y}\right) \\
& -\bar{F}\left[\frac{1}{\left[\bar{g}_{0}+\bar{x} \sin \theta\right]^{\circ 2}}+\frac{2 \vec{y}}{\left[\bar{g}_{0}+\bar{x} \sin \theta\right]^{\circ 3}}+\frac{3[\vec{y}]^{\circ 2}}{\left[\bar{g}_{0}+\bar{x} \sin \theta\right]^{\circ 4}}+\frac{4[\vec{y}]^{\circ 3}}{\left[\bar{g}_{0}+\bar{x} \sin \theta\right]^{\circ 5}}+\cdots\right], \\
\frac{\partial \varphi\{\vec{y}\}}{\partial \vec{y}}= & w_{i j}^{(4)} \diamond \bar{h}^{3}(\bar{x})+2 w_{i j}^{(3)} \diamond \frac{d \bar{h}^{3}(\bar{x})}{d \bar{x}}+w_{i j}^{(2)} \diamond \frac{d^{2} \bar{h}^{3}(\bar{x})}{d \bar{x}^{2}} \\
& -\bar{F}\left[\frac{2 I_{u}}{\left[\bar{g}_{0}+\bar{x} \sin \theta\right]^{\circ 3}}+\frac{6 I_{u} \diamond[\vec{y}]}{\left[\bar{g}_{0}+\bar{x} \sin \theta\right]^{\circ 4}}+\frac{12 I_{u} \diamond[\vec{y}]^{\circ 2}}{\left[\bar{g}_{0}+\bar{x} \sin \theta\right]^{\circ 5}}+\frac{20 I_{u} \diamond[\vec{y}]^{\circ 3}}{\left[\bar{g}_{0}+\bar{x} \sin \theta\right]^{\circ 6}}+\cdots\right] .
\end{aligned}
$$

Eqs. (2.10a) and (2.10b) reveal that the iteration formulation of the Newton-Raphson method in this case is

$$
\vec{y}^{(k+1)}=\vec{y}^{(k)}-\frac{\varphi\left\{\vec{y}^{(k)}\right\}}{\frac{\partial \varphi\left\{\vec{y}^{(k)}\right\}}{\partial \vec{y}}} .
$$

Notably, the linear solution for a cantilever beam with a uniformly distributed load is used as the initial estimate in the iterative procedure.

\section{Numerical analyses of various micro-tweezers}

\subsection{Example 1: Cantilever rectangular beam micro-tweezers}

This work first simulates the uniform rectangular cantilever beam micro-tweezers addressed in the references [1-3] to confirm the accuracy and feasibility of the proposed DQM approach. The current simulation results are then compared with those presented in the relevant literature. 
Example 3.1 (Simplified micro-tweezers [3]). In [3], simplified rectangular beam microtweezers with $\theta=0^{\circ}$, as depicted in Fig. 1, are investigated by applying a hybrid BEM and FEM method. In the current example, the beam length $L_{b}$ is $200 \mu \mathrm{m}$; Young's modulus for the tungsten beam material is $E=410 \mathrm{GPa}$ and the beam width is $b=2.7 \mu \mathrm{m}$. Additionally, the thickness of the rectangular beam is $h_{0}=2.9 \mu \mathrm{m}$ and the initial gap is $d_{0}=2 g_{0}=4 \mu \mathrm{m}$. Table 1 summarizes the beam geometry. Fig. 2 reveals that the gap distance change $\left(2 y_{e}\right)$ between two micro-arm tips declines gradually as the applied voltage is increased toward a critical value. The parameter ye denotes the tip deflection for each arm of the microtweezers. Beyond this critical voltage, the two cantilever micro-arms are pulled suddenly toward each other. Good agreement exists between the pull-in voltage of $79.6 \mathrm{~V}$ calculated by the proposed DQM and the pull-in voltage of 78V reported in 1995 by F. Shi et al., which they obtained using the hybrid FEM-BEM method [3]. The difference between the two sets of results is found to be under $2 \%$.

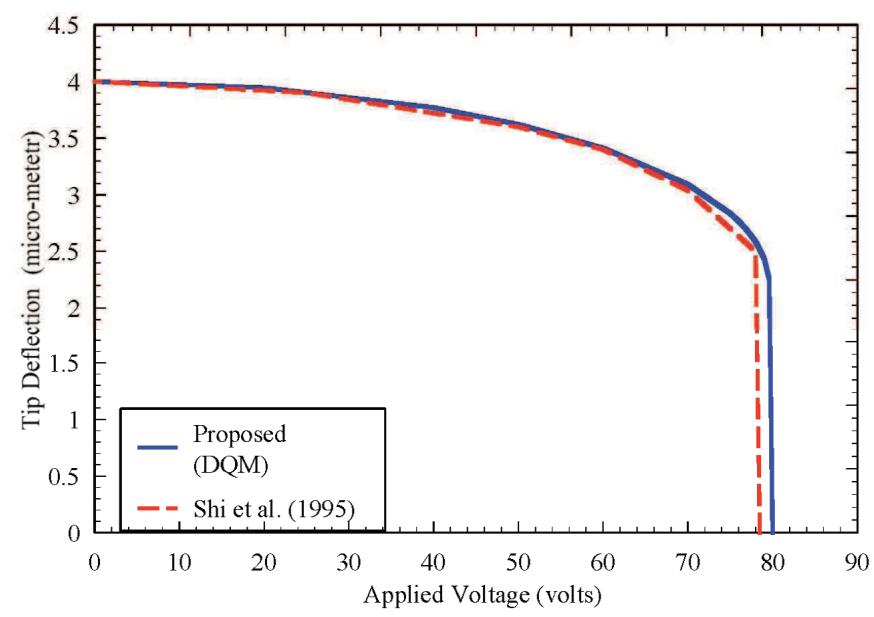

Figure 2: Variation in the tip gap of electrostatic micro-tweezers.

Table 1: The dimension of the cantilever beam-type micro-tweezers.

\begin{tabular}{||c|c|c|c|c|c||}
\hline Example & $\theta$ & $L_{b}(\mu m)$ & $d_{0}(\mu m)$ & $h_{0}(\mu m)$ & Material/Young's modulus $(E)$ \\
\hline 1.1 & $0^{\circ}$ & 200 & 4 & 2.9 & Tungsten / 410 (GPA) \\
\hline 1.2 & $0.5^{\circ}$ & 200 & 3 & 2.7 & Tungsten / 410 (GPA) \\
\hline 1.3 & $0.5^{\circ}$ & 200 & 3 & $3.1^{*}$ & $\begin{array}{c}\text { An effective combination of Tungsten } \\
\text { and silicon dioxide coating/410 (GPA) /73 (GPA) }\end{array}$ \\
\hline
\end{tabular}

*: The beam thickness of Tungsten is $2.7 \mu \mathrm{m}$ with the thickness $0.2 \mu \mathrm{m}$ of $\mathrm{SiO}_{2}$ on both sides.

\section{Calculating contact forces}

When the applied voltage reaches the critical pull-in voltage, the tips of microtweezers contact the object. The contact forces increase as the applied voltage is increased 


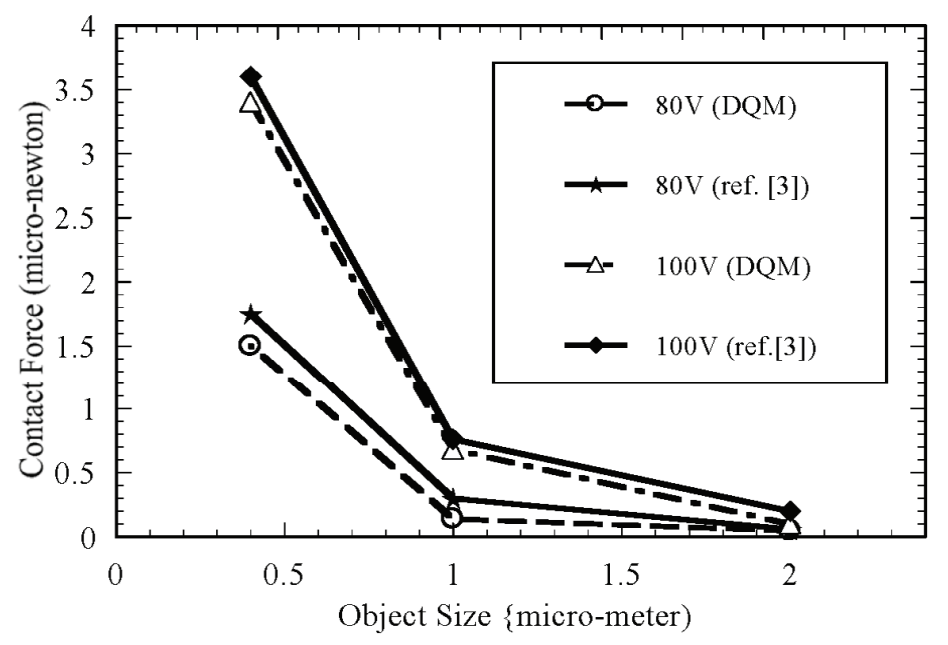

Figure 3: Variation in contact force for different object sizes.

further. The assumption micro-tweezers' arms are assumed to be symmetrical, so a contact force applies along the centerline of the gap between the two arms.

This study considers a simple compressive spring between the free ends of the microtweezers arms to model the effect of the two arm tips' contacting the gripped object. Therefore, when the tips are in contact with the object, the beam is no longer cantilevered, but is considered to be spring-supported at the tip. This modification can be made by inserting the spring force at the tip of the micro-arm as a boundary condition. This spring force $f_{s}$ changes the boundary conditions in the original set of conditions (2.3). Accordingly, the shear force at the end of the micro-arm beam equals the spring force:

$$
E \operatorname{Iy}^{\prime \prime \prime}(x)=f_{s} .
$$

The contact force can then be determined by multiplying the stiffness matrix of the beam by the deflection calculated from the tip support. Fig. 3 compares the contact forces calculated by the DQM with the values predicted by the hybrid BEM and FEM approach [3]. The two sets of results are similar and mutually consistent.

Example 3.2 (Micro-tweezers without silicon dioxide coating [1-3]). In [1,2], CVD tungsten micro-tweezers were fabricated and investigated. Their, two micro-arm beams were rigidly attached at the fixed end with $\theta=0.5^{\circ}$. The other dimensions were as shown in Table 1. The experimental results reveals that the pull-in voltage was $125 \mathrm{~V}$ [1,2]. In contrast, the proposed DQM approach predicts a pull-in voltage of $121.5 \mathrm{~V}$, while the result obtained using the hybrid FEM-BEM method was 112V. Fig. 4 compares the tip deflection behaviors of the micro-arm as calculated by the presented method, with those obtained by the other two reported methods. Fig. 5 shows the variation in micro-arm deflection against arm length, at various applied voltages. 


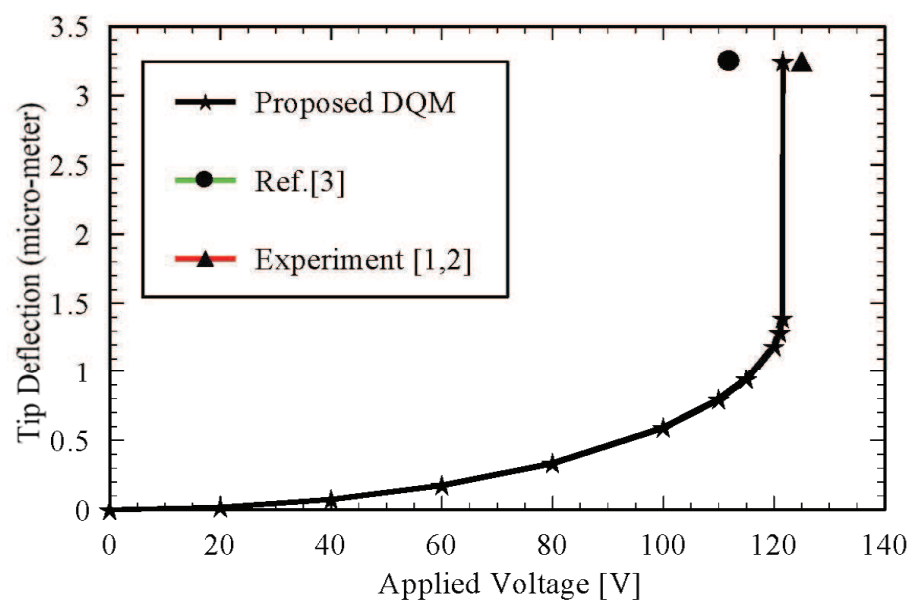

Figure 4: Variation in tip deflection for micro-tweezers with different driving voltages.

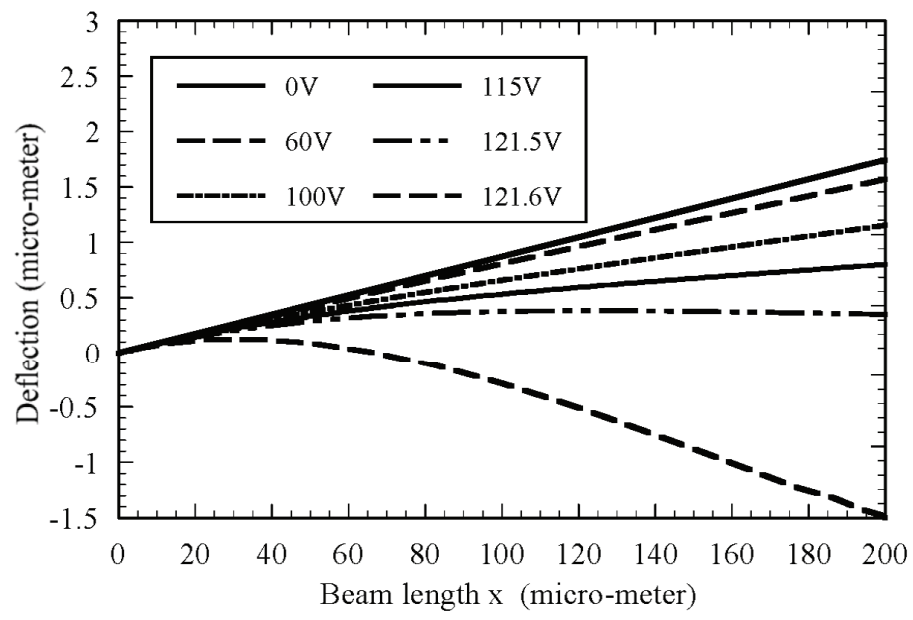

Figure 5: Beam deflection shapes at different applied voltages.

Example 3.3 (Micro-tweezers with silicon dioxide coating [1-3]). MacDonald et al. [1] and Chen et al. [2] produced micro-tweezers coated with a silicon dioxide film to prevent electric shorting when the two beams were pulled together. The thickness of the silicon dioxide was $0.2 \mu \mathrm{m}$ and the beam length $L_{b}$ was $200 \mu \mathrm{m}$. Moreover, Young's modulus of the tungsten beam material was $E=410 \mathrm{GPa}$, while that of the silicon dioxide was $E_{s}=73 \mathrm{GPa}$. Fig. 6(a) presents the cross-sectional view of the composite micro-beam. In this work, a 2-D model is used to model the composite beam, and the assumption is made that "planar sections remain planar" [3], as shown in Fig. 6(b). Hence, the effective EI of the composite micro-beam equals the summation of its tungsten and silicon dioxide com- 


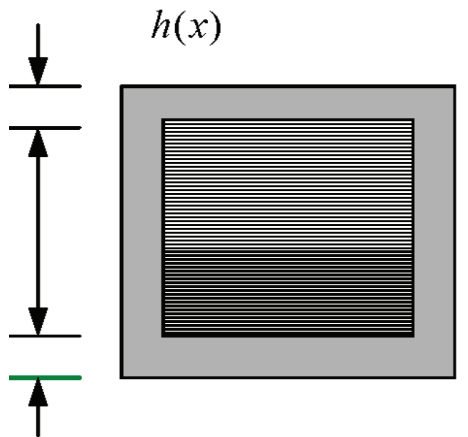

(a)

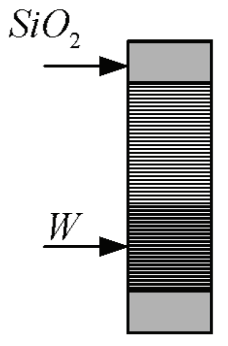

(b)

Figure 6: The cross section of a silicon dioxide coated micro-beam.

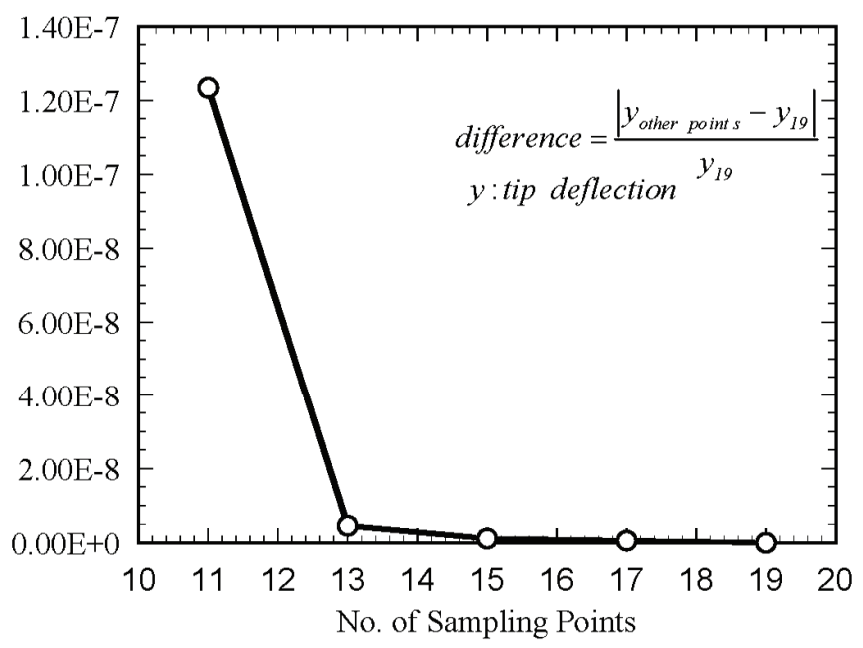

Figure 7: Convergence of the calculated tip deflection with different number of sampling points.

ponents, so $E I=E I_{1, S_{i} O_{2}}+E I_{2, \text { tungsten }}+E I_{3, S_{1} \mathrm{O}_{2}}$. The result of the pull-in voltage obtained by the DQM approach was $141.5 \mathrm{~V}$, which can be compared to an experimental value of $150 \mathrm{~V}[1,2]$, and the value 157 160V obtained using the FEM-BEM hybrid method [3]. Importantly, the proposed DQM algorithm depends on the use of just 19 sampling points along the beam and can converge with a tolerance of $10^{-8}$ in four iterations. Fig. 7 plots the convergence tendency of the tip deflections calculated using the DQM for various numbers of selected sampling points. The locations of the sampling points along the beam follow according to the Chebyshev-Gauss-Lobatto distribution [23]

$$
\bar{x}_{i}=\frac{1}{2}\left[1-\cos \frac{(i-1)}{(N-1)} \pi\right], \text { for } i=1,2, \cdots, N .
$$


These comparisons between the rectangular cantilever beam micro-tweezers herein with those in the literatures reveal excellent agreement between DQM and other reference results. The comparisons presented above for rectangular cantilever beam micro-tweezers verify that the simulation results obtained using the proposed DQM approach agree closely with the results in the literature. The critical applied voltage, which results in the pull-in effect, is known to depend on the particular shape of the micro-arm. The following section investigates and exploits this fact.

\subsection{Shaped cantilever beam micro-tweezers}

In this section, the beam length, width and material properties, are as presented previously in Example 3.3. However, the beam thickness profile is different. As presented in Fig. 1(b), the beam thickness, $\bar{h}(\bar{x})$, has a shape order of $m$, and its thickness at its two ends is $h_{0}$ and $h_{1}$. Table 2 summarizes the dimensions in this particular example. Fig. 8 compares the tip deflections of the original rectangular beam at various applied voltages with the corresponding tip deflections of a tapered beam $(m=1)$ and second order poly-

Table 2: The dimension of micro-tweezers with different shape profiles.

\begin{tabular}{||c|c|c|c|c|c|c||}
\hline Example 2 & $m$ & $\theta$ & $L_{b}(\mu m)$ & $d_{0}(\mu m)$ & $h_{0}(\mu m)$ & $h_{1}(\mu m)$ \\
\hline Ex215 & 1 & $0.5^{\circ}$ & 200 & 3 & 3.1 & 1.5 \\
\hline Ex225 & 2 & $0.5^{\circ}$ & 200 & 3 & 3.1 & 1.5 \\
\hline Ex223 & 2 & $0.5^{\circ}$ & 200 & 3 & 3.1 & 0.3 \\
\hline
\end{tabular}

*: The effective Young's modulus $E$ is a combination of the Tungsten 410(GPA) and the silicon dioxide coating 73(GPA).

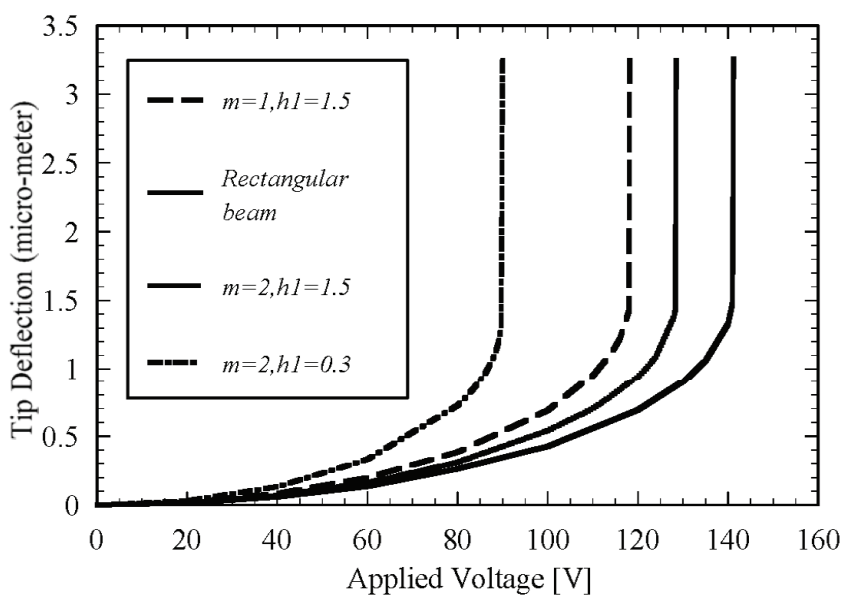

Figure 8: Variation in tip deflection for the micro-arm with different beam shape profiles. 
nomial $(m=2)$ shaped beams. The critical pull-in voltage of the original rectangular beam is $141.2 \mathrm{~V}$. The value is lower, $128.5 \mathrm{~V}$, for shaped beam with $m=2$ and $h_{1}=1.5 \mu m$, and is even lower, $118.2 \mathrm{~V}$, for the beam of taper-shaped $(m=1)$ and $h_{1}=1.5 \mu \mathrm{m}$. The lowest pullin voltage of $87.2 \mathrm{~V}$ is achieved for a beam with shape factors of $m=2$ and $h_{1}=0.3 \mu \mathrm{m}$. The results verify that a designer can control the range of pull-in voltages of micro-tweezers by specifying the shape profiles of the beams.

\section{Conclusions}

This work applied the DQM approach to study the tip deflection and pull-in voltages for variously shaped cantilever beam micro-tweezers. The numerical results demonstrated that the proposed DQM technique constitutes an accurate and efficient means of simulating the deflection and the clamp force responses of nonlinear micro-tweezers. A comparison of the results obtained from the proposed DQM approach with those reported in the literature reveals the following:

1. The DQM approach provides efficient and accurate simulations of the pull-in voltage for variously shaped beam-type electrostatic micro-tweezers. The method depends on the just 19 sampling points to yield a convergent result.

2. The proposed DQM approach can be used to calculate efficiently and accurately not only the nonlinear deflection of a simple rectangular beam, but also those of shaped cantilever beam micro-tweezers. Therefore, the DQM is an appropriate and convenient means of designing and analyzing electrostatic micro-tweezers.

3. The results reveal that a properly shaped micro-arm can reduce the pull-in voltage from $141 \mathrm{~V}$ (for a rectangular micro-beam) to $87 \mathrm{~V}$ (for a shaped micro-beam with $m=2$ and $\left.h_{1}=0.3 \mu m\right)$.

\section{Acknowledgments}

The financial support by the Ministry of Science and Technology, TAIWAN, through Grant MOST 105-2221-E-230-004 of the Cheng Shiu University is gratefully acknowledged.

\section{References}

[1] N. C. MacDonald, L. Y. Chen, J. J. Yao, Z. L. Zhang, J. A. Mcmillan, D. C. Thomas and K. R. Haselton, Selective chemical vapor deposition of tungsten for microelectromechanical structures, Sens. Actuators, 20 (1989), 123-133.

[2] L. Y. Chen, Z. L. Zhang, J. J. Yao, D. C. Thomas and N. C. MacDonald, Selective chemical vapor deposition of tungsten for microdynamic structures, Proc. IEEE, (1989), 82-87. 
[3] F. Shi, P. Ramesh and S. Mukherjee, Simulation methods for micro-electro-mechanical structures (MEMS) with application to a microtweezer, Comput. Struct., 56(5) (1995), 769-783.

[4] K. E. Petersen, Silicon as a mechanical material, Proc. IEEE, 70(5) (1982), 420-457.

[5] G. M. Koppelman, OYSTER, A three-dimensional structural simulator for microelectromechanical design, Sens. Actuators., 20 (1989), 179-185.

[6] R. H. Price, J. E. Wood and S. C. Jacobsen, Modeling considerations for electrostatic forces in electro-static microactuators, Sens. Actuators, 20 (1989), 107-114.

[7] S. D. Senturia, R. M. Harris, B. P. Johnson, Kim Songmin, K. Nabors, M. A. Shulman and J. K. White, A computer-aided design system for microelectromechanical systems (MEMCAD), J. Microelectromech. Syst., 1(1) (1992), 3-13.

[8] J. R. Gilbert, P. M. Osterberg, R. M. Harris, D. O. Ouma, X. Cai, A. Pfajfer, J. White and S. D. Senturia, Implementation of a MEMCAD system for electrostatic and mechanical analysis of complex structures from mask descriptions, Proc. IEEE, (1993), 207-212.

[9] P. M. Osterberg, H. Yie, X. Cai, J. White and S. D. Senturia, Self-consistent simulation and modeling of electrostatically deformed diaphragms, Proc. IEEE, (1994), 28-32.

[10] P. M. Osterberg and S. D. Senturia, M-Test: a test chip for MEMS material property measurement using electrostatically actuated test structures, J. Microelectromech. Syst., 6(2) (1997), 107-117.

[11] R. Legtenberg, J. Gilbert, S. D. Senturia, and M. Elwenspoek, Electrostatic curved electrode actuators, J. Microelectromech. Syst., 6(3) (1997), 257-265.

[12] M. A. Gretillat, Y. J. Yang, E. S. Hung, V. Rabinvich, G. K. Ananthasuresh, N. F. Rooij and S. D. Senturia, Nonlinear electromechanical behavior of an electrostatic microrelay, Proc. IEEE, (1997), 1141-1144.

[13] E. S. Hung and S. D. Senturia, Extending the travel range of analog-tuned electrostatic actuators, J. Microelectromech. Syst., 8(4) (1999), 497-505.

[14] B. E. Volland, H. Heerlein and I. W. Rangelow, Electrostatically driven microgripper, Microelectron. Eng., 61-62 (2002), 1015-1023.

[15] V. Seidemann, S. Bütefisch and S. Büttgenbach, Fabrication and investigation of in-plane compliant SU8 structures for MEMS and their application to micro valves and micro grippers, Sens. Actuators. A, 97-98 (2002), 457-461.

[16] O. Fuchiwaki, A. Ito, D. Misaki and H. Aoyama, Multi-axial micromanipulation organized by versatile micro robots and micro tweezers, Proc. IEEE International Conference on Robotics and Automation Pasadena, (2008), 19-23.

[17] L. Sujatha, N. Vigneswaran and S. Mohamed Yacin, Design and analysis of electrostatic micro tweezers with optimized hinges for biological applications using coventor ware, Procedia Eng., 64 (2013), 283-291.

[18] B. E. Vollanda, K. Ivanovab, Tzv. Ivanova, Y. Sarovb, E. Guliyeva, A. Persaudb, J. P. Zöllnera, S. Kletta, I. Kosticd and I. W. Rangelowa, Duo-action electro thermal micro gripper, Microelectron. Eng., 84(5C8) (2007), 1329-C1332.

[19] A. Deutschinger, U. Schmid, M. Schneider, W. Brenner, H. Wanzenböck, B. Volland, Tzv. IvanovI and W. Rangelow, Characterization of an electro-thermal micro gripper and tip sharpening using FIB technique, Microsyst. Technol., 16(11) (2010), 1901-1908.

[20] R. E. Bellman, B. G. Kashef and J. Casti, Differential quadrature: a technique for the rapid solution of nonlinear partial equations, J. Comput. Phys., 10 (1972), 40-52.

[21] Y. Feng and C. W. Bert, Application of the quadrature method to flexural vibration analysis of a geometrically nonlinear beam, Nonlinear Dyn., 3 (1992), 13-18.

[22] X. Wang and C. W. Bert, A new approach in applying differential quadrature to static and 
free vibration analysis of beams and plates, J. Sound Vib., 162(3) (1993), 566-572.

[23] C. W. Bert and M. Malik, Free vibration analysis of tapered rectangular plates by differential quadrature method: a semi-analytical approach, J. Sound Vib., 190(1) (1996), 41-63.

[24] M. Malik and C. W. Bert, Implementing multiple boundary conditions in the DQ solution of high-order PDE's: application to free vibration of plates, Int. J. Numer. Methods Eng., 39 (1996), 1237-1258.

[25] W. Chen and T. Zhong, The study on the nonlinear computations of the DQ and DC methods, J. Numer. Methods Partial Difference Equations, 13 (1997), 57-75.

[26] W. Chen, C. Shu, W. He and T. Zhong, The application of special matrix product to differential quadrature solution of geometrically nonlinear bending of orthotropic rectangular plates, Comput. Struct., 74 (2000), 65-76.

[27] S. Tomasiello, Differential quadrature method: application to initial-boundary-value problems, J. Sound Vib., 218(4) (1998), 573-585.

[28] T. Y. Wu and G. R. Liu, The generalized differential quadrature rule for initial-value differential equations, J. Sound Vib., 233(2) (2000), 195-213.

[29] S. Tomasiello, Simulating non-linear coupled oscillators by an iterative differential quadrature method, J. Sound Vib., 265 (2003), 507-525.

[30] T. Y. Wu, G. R. Liu and Y. Y. Wang, Application of the generalized differential quadrature rule to initial-boundary-value problems, J. Sound Vib., 264 (2003), 883-891. 